\title{
Serious Complication of Anticoagulation in Vessel Thrombosis-associated Behçet Disease
}

\author{
Kotaro Ouchi ${ }^{1}$, Ryosuke Narui ${ }^{2}$, Toru Sakuma ${ }^{1}$ and Hiroya Ojiri ${ }^{1}$
}

\begin{abstract}
:
Anticoagulation in venous thrombosis associated with Behçet disease (BD) is controversial. We herein report a 47-year-old woman with vessel thrombosis and pulmonary artery aneurysm (PAA) associated with BD who died of massive hemoptysis under oral anticoagulant. Although she was initially diagnosed with oral contraceptive-induced venous thromboembolism, a subsequent investigation led to a diagnosis of BD. Followup computed tomography (CT) showed persistent thrombus, so anticoagulant was continued for persistent thrombus. She died of massive hemoptysis after the development of PAA was identified on follow-up CT during the period of anticoagulation. Great care to prevent bleeding events is required when administering anticoagulants for BD with vessel thrombosis.
\end{abstract}

Key words: anticoagulation, Behçet disease, hemoptysis, pulmonary artery aneurysm, vessel thrombosis

(Intern Med 59: 1615-1620, 2020)

(DOI: 10.2169/internalmedicine.4109-19)

\section{Introduction}

Although the diagnosis of Behçet disease (BD) is made based on clinical findings, the clinical phenotypes of $\mathrm{BD}$ is extremely diverse aside from classic manifestations, such as oral and genital aphthous or ulcerative lesions. Therefore, it is difficult for clinicians not familiar with BD to correctly attribute vascular manifestations, including major vessel thrombosis, to $\mathrm{BD}$ itself.

Anticoagulation is the standard treatment of systemic venous thrombosis of any etiology (1). However, the usage of anticoagulants to treat venous thrombosis due to BD is controversial and a matter of great debate between experts (2). There are no large controlled studies available regarding the best approach to managing $\mathrm{BD}$ patients complicated by thrombosis and the exact role of long-term anticoagulation in these cases. In addition, clear and definite data on the best approach to manage pulmonary artery aneurysm (PAA), which is a life-threatening complication of $\mathrm{BD}$, is also lacking.

The unfortunate outcome of the present case relates to the role of oral anticoagulation for the treatment of BD-related venous complications. We report this case in order to highlight the serious dilemma associated with administering anticoagulant treatment for major vessel thrombosis in BD patients and consider how best to approach to PAA complicated with BD.

\section{Case Report}

A 47-year-old woman with no significant medical history presented with hemosputum. The patient had reported a recurrent fever and cough with chest pain for the past month. She smoked 15 cigarettes per day and drank socially. There was no significant family history. Her only regular medication was a contraceptive pill.

On a physical examination, her body temperature was $37.1^{\circ} \mathrm{C}$, blood pressure was $123 / 81 \mathrm{mmHg}$, pulse $101 / \mathrm{min}$, respiratory rate $16 / \mathrm{min}$ and $\mathrm{O}_{2}$ saturation $97 \%$ on room air. Her legs were swollen. The remainder of the physical examination was unremarkable.

Laboratory data on admission showed a markedly elevated white blood cell count $(15,200 / \mu \mathrm{L})$, platelet count $(557,000 / \mu \mathrm{L})$, C-reactive protein $(\mathrm{CRP})$ level $(14.3 \mathrm{mg} / \mathrm{dL})$ and fibrinogen level $(740 \mathrm{mg} / \mathrm{dL})$. The D-dimer levels $(2.2$

${ }^{1}$ Department of Radiology, The Jikei University School of Medicine, Japan and ${ }^{2}$ Department of Cardiology, The Jikei University School of Medicine, Japan

Received: October 27, 2019; Accepted: February 4, 2020; Advance Publication by J-STAGE: March 26, 2020

Correspondence to Dr. Kotaro Ouchi, kotaro-alex@jikei.ac.jp 


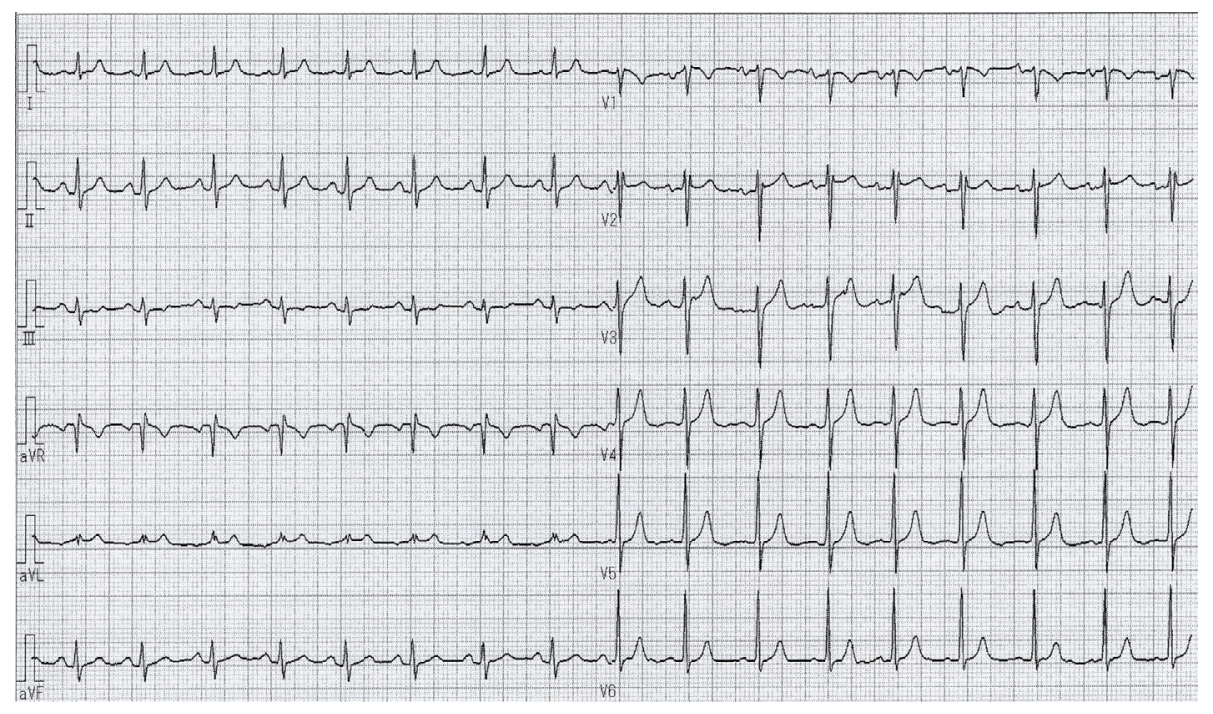

Figure 1. An ECG on admission showed sinus tachycardia.

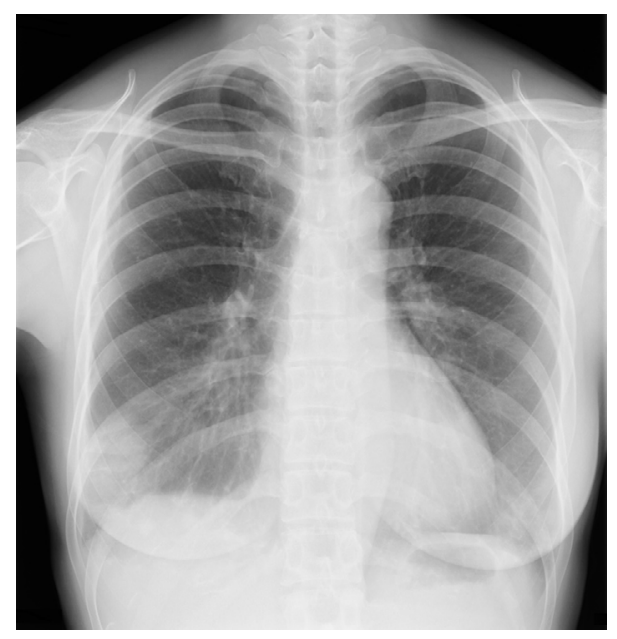

Figure 2. Chest $\mathbf{X}$-ray showed infiltration in bilateral lower lung fields.

$\mu \mathrm{g} / \mathrm{mL})$ were also elevated, while serum albumin levels (3.1 $\mathrm{mg} / \mathrm{dL}$ ) were slightly low. The levels of antithrombin, protein $\mathrm{C}$, protein $\mathrm{S}$ and lupus anticoagulant were normal. An electrocardiogram revealed sinus tachycardia (Fig. 1). Chest $\mathrm{X}$-ray showed infiltration in the bilateral lower lung fields (Fig. 2).

Under suspicion of underlying pulmonary diseases, including pulmonary thromboembolism (PTE), contrast computed tomography (CT) of the thorax, abdomen, pelvis and lower limbs was performed, revealing a filling defect compatible with thrombosis in the pulmonary artery and deep leg vein. CT also showed wedge-shaped or linear lesions which suggested the presence of infarction (Fig. 3). Echocardiography showed no findings of right heart dilatation, and the peak tricuspid regurgitation velocity was estimated to be $259 \mathrm{~cm} / \mathrm{s}$, which indicates a low probability of pulmonary hypertension.

Inherited thrombophilia was excluded based on laboratory data and her medical history, so a diagnosis of drug-induced deep vein thrombosis (DVT) due to her contraceptive pill and pulmonary infarction complicated with pulmonary embolism was made. She was admitted to our hospital, the contraceptive pill was discontinued, and treatment with heparin and warfarin was initiated.

She was discharged with an international normalized ratio of prothrombin time (PT-INR) of 2.4 within the target range but presented again 3 days later with recurrent hemoptysis. On readmission, her PT-INR was markedly elevated at 4.3. The dose of warfarin was reduced, and PT-INR was controlled within the target range at 1.6.

She was referred for panuveitis with mild elevation of the CRP levels after three months. A final diagnosis of incomplete $\mathrm{BD}$ was made following an investigation based on the history of vessel thrombosis, panuveitis and aphthous stomatitis, and colchicine was initiated orally. The oral anticoagulant was changed to edoxaban from warfarin due to its complexity of blood concentration monitoring and dosing which may increase the risk of bleeding, although the PT-INR was stable within the target range. The D-dimer level was also within the normal range. Although mild elevation of the white blood cell count and CRP level persisted, she was stable except for episodes of transient and mild hemoptysis (Fig. 4). The thrombus persisted on follow-up CT at three months after readmission, so anticoagulant therapy was continued.

Follow-up CT nine months later showed persistent thrombus and the development of a new PAA in the right lower lobe (Fig. 5). Two weeks after follow-up CT, the patient died of massive hemoptysis. An autopsy revealed diffuse alveolar hemorrhaging to be the cause of death. However, a causal relationship between the alveolar hemorrhaging and PAA was not proven.

\section{Discussion}

We identified two clinical issues. First, it is difficult to 

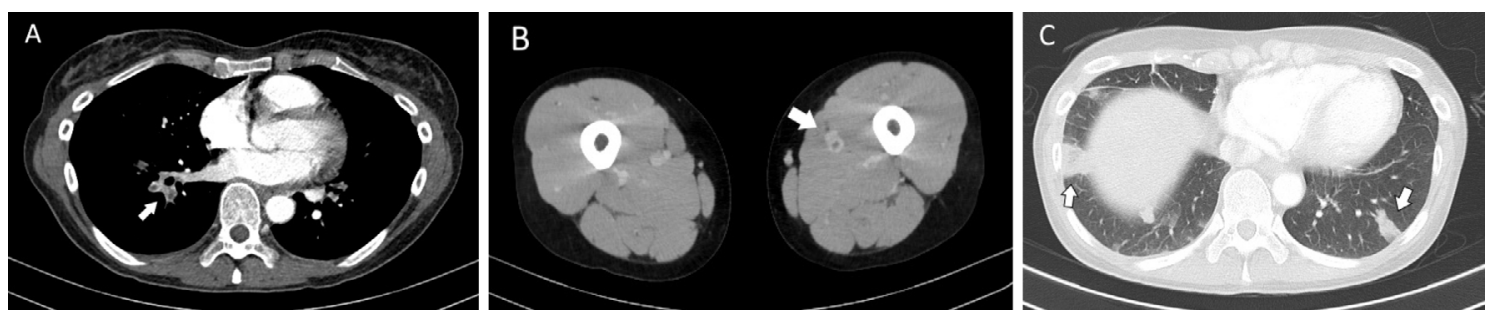

Figure 3. Contrast CT showed a filling defect compatible with thrombosis in the pulmonary artery (arrow; A) and deep leg vein (arrow; B) along with wedge-shaped, linear lesions that were characteristic for infarction in the lung fields (arrows; $\mathrm{C}$ ).
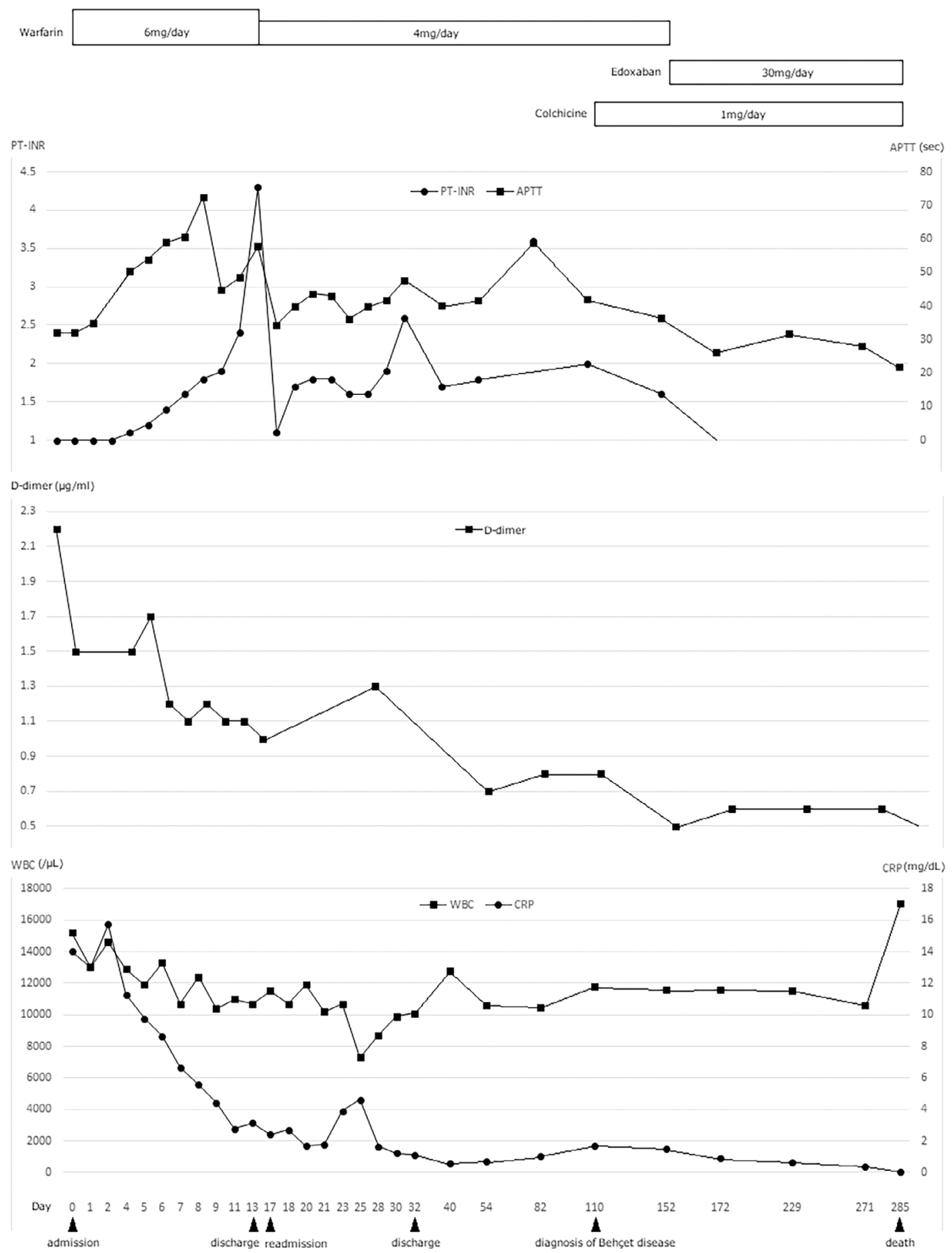

Figure 4. The patient's clinical course. 


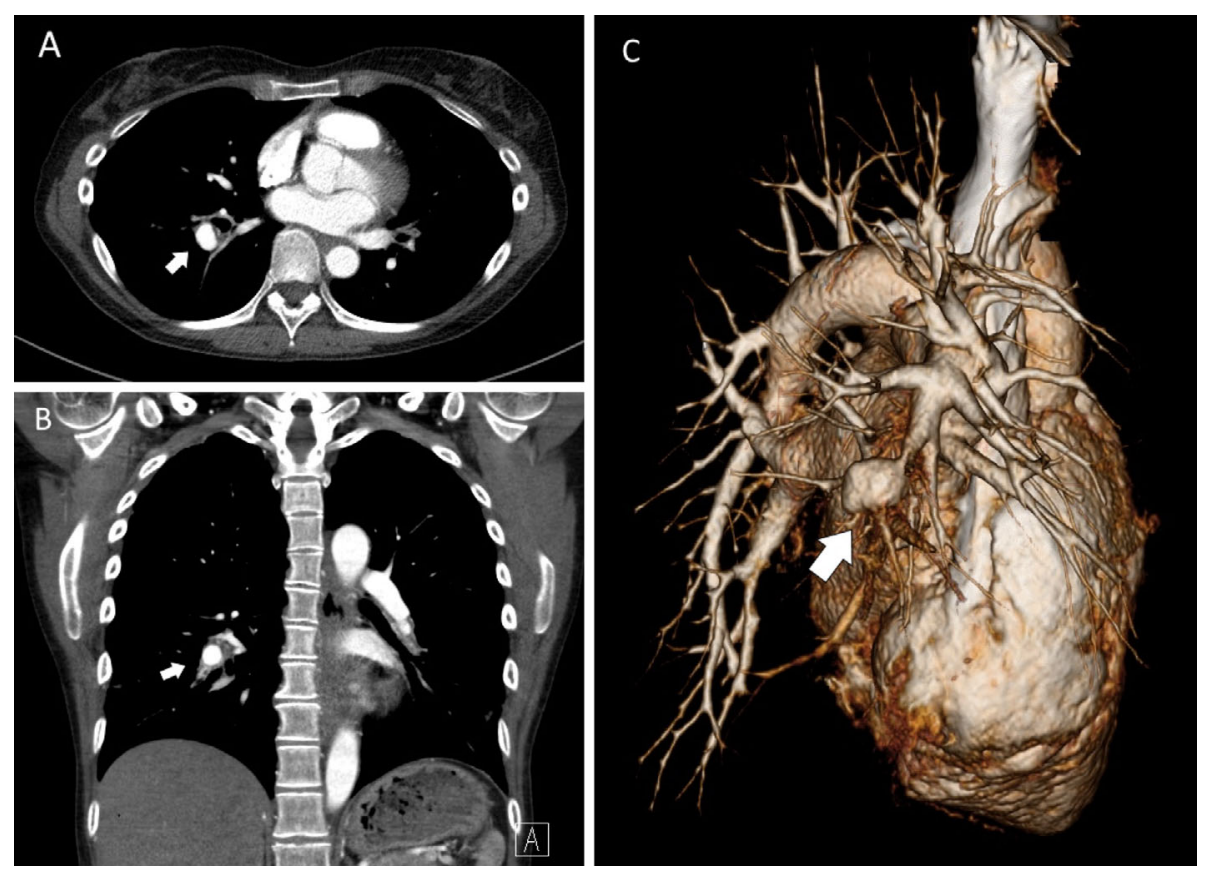

Figure 5. Contrast CT axial (arrow A), coronal (arrow B) and volume rendering (arrow C) images obtained nine months later showed the development of a new pulmonary arterial aneurysm in the right lower lobe.

correctly attribute vascular manifestations, including major vessel thrombosis, to BD itself, especially when there is another etiology with a higher likelihood of being responsible, as in this case. Second, great care to prevent bleeding events is required when administering anticoagulants for pulmonary artery and DVT in BD. Regarding the present case, the cause of death and massive hemoptysis under anticoagulant therapy was not found to be related to the PAA.

The misdiagnosis of hemoptysis as being a result of pulmonary infarction associated with PTE can delay the diagnosis. There are no pathognomonic diagnostic laboratory tests in $\mathrm{BD}$, so the diagnosis is made based on clinical findings. The clinical phenotypes of BD are extremely diverse apart from classic manifestations, such as oral and genital aphthous or ulcerative lesions. Therefore, it is difficult for clinicians not familiar with BD to correctly attribute vascular manifestations to $\mathrm{BD}$ itself. In addition, venous thrombosis in $\mathrm{BD}$ occurs more frequently during active disease in men than in women (3). The present patient was initially diagnosed with drug-induced venous thromboembolism because of her history of regularly using a contraceptive pill and the sex-based prevalence. Although there was a possibility of both drug-induced DVT and thrombus associated with $\mathrm{BD}$ coexisting at the first visit, $\mathrm{BD}$ alone was more likely to blame for the vessel thrombus, considering her clinical course. The possibility of BD should be considered for patients with a history of recurrent hemoptysis and venous thrombosis. This will lead to an early diagnosis and appropriate therapeutic intervention for patients with $\mathrm{BD}$, which is crucial for improving their prognosis.

The application of anticoagulant therapy for $\mathrm{BD}$, which may have grave consequences, requires great care to manage bleeding events. A previous report showed that the administration of direct oral anticoagulants to high-risk antiphospholipid syndrome patients with venous thromboembolism (VTE) was associated with an excess of major bleeding events compared with warfarin (4). Retrospectively, there might have been grounds to consider switching from edoxaban to warfarin again in the present case. Venous disease in BD is believed to result from endothelial inflammation leading to thrombosis, so the best approach to prevent venous thrombotic events in BD may be controlling systemic inflammation rather than administering primary anticoagulation (5). Some reports have suggested that thrombotic events should be treated with anticoagulation using standard approaches if they occur (6). However, recent retrospective studies have shown that the risk of DVT is lower in patients treated with immunosuppressive agents than in those only receiving anticoagulants (7-9). The recurrence of thrombotic events or even an increase in thrombus size after the administration of warfarin or heparin therapy has still been documented (10). A previous report found that venous thrombosis in BD is due to an inflammatory process and that pulmonary artery occlusion in BD also differs from classic PTE disease, typically representing in situ thrombi complicating an underlying vasculitis rather than emboli (11). In addition, the thrombus formed adheres tightly to the vessel wall, necessitating the use of anti-inflammatory agents only (12). The possibility of bleeding after the rupture of a coexisting aneurysm anywhere in the body may also have detrimental consequences due to anticoagulation treatment $(13,14)$. In our case, the thrombus persisted despite anticoagulation, which strengthens the view that inflammation rather than thrombophilic factors plays a role in the pathogenesis of 
vascular complications in BD. It may therefore have been more appropriate to consider discontinuing anticoagulant therapy rather than continuing anticoagulation for persistent thrombus associated with $\mathrm{BD}$.

Current guidelines recommend anticoagulation treatment for more than 3 months after the first episode of VTE associated with persistent risk factors $(15,16)$. However, the decision to continue or stop anticoagulants beyond the first three months is made by weighing the risk of recurrent VTE and bleeding during ongoing treatment. Therefore, we must identify patients with an increased bleeding risk while on chronic anticoagulation treatment. Clinical tools for predicting both the thrombotic and bleeding risk over the long term, such as the VTE-BLEED score, may be helpful for identifying the minority of patients who require closer attention than others while on anticoagulation after VTE and for making decisions regarding long-term anticoagulant treatment (17).

PAA in the presence of systemic thrombosis is a rare manifestation almost unique to BD. The term "Incomplete Behçet's Disease" has also been used for Hughes-Stovin Syndrome (HSS) patients due to the similarities between these two disorders of unknown etiology, and the pulmonary system involvement in $\mathrm{BD}$ can be identical to that in HSS (18). These aneurysms are life-threatening complications of $\mathrm{BD}$, and the bleeding that follows their rupture may lead to death in patients under anticoagulation treatment. However, the autopsy in the present patient showed no evidence of a relationship between the patient's massive hemoptysis and PAA.

The most common clinical manifestation of pulmonary involvement in $\mathrm{BD}$ is hemoptysis, which is due to a bronchoarterial fistula and may occur with or without an aneurysm (19). The massive hemoptysis in the present patient may have been caused by this bronchoarterial fistula rather than a PAA. Arterial aneurysms in BD occur secondary to obliterative endarteritis of the vasa vasorum (20). These changes in the vasa vasorum lead to ischemic changes in the vessel wall, which in turn lead to the loss of elastic fibers and of muscle cells in the media layer or to transmural necrosis ending in the formation of true aneurysms. If all of these structural changes result in the disruption of the anatomical integrity of the vessel wall, a pseudo-aneurysm is formed. It is not uncommon to see a true and pseudoaneurysm in BD side by side (20). However, it is difficult to distinguish between the two on imaging.

The three main treatment options for PAA associated with $\mathrm{BD}$ are immunosuppressive treatment, surgical resection and embolization. Surgical experience in both emergency and selective settings for PAA has been disappointing (21). There is also a $25 \%$ risk of recurrent aneurysms in BD patients, usually at the anastomosis site after surgery (22). Transcatheter embolization of PAA has been performed in BD patients as an alternative approach (23-25). However, no long-term follow-up data exist. Transcatheter embolization might not be the best management option in $\mathrm{BD}$, as the pathological involvement is mainly in the surrounding invasive structures of the aneurysm, not the aneurysm itself. Furthermore, if PAA is found to be a pseudo-aneurysm, then performing embolization with coil packing would be inappropriate for such a pseudo-aneurysm, since they tend to be vulnerable regions because of lack of all three normal layers of the arterial wall, and thus they pose a high risk for intraoperative bleeding and rebleeding.

However, a previous report described cases in which PAAs disappeared or decreased in size in BD patients receiving immunosuppressive therapy for PAA (26). In the present case, the development of a new PAA suggested the progression of vascular inflammation. Although the CRP levels were decreasing, there may have been a discrepancy between the CRP levels and the disease activity, as seen in systemic lupus erythematosus and some cases of active rheumatoid arthritis $(27,28)$. In these cases, the erythrocyte sedimentation rate (ESR) which is one of the most widely used indicators of the acute phase response might have been a better marker for inflammation than the CRP. Unfortunately, the ESR was not monitored in our case. Complementary use of both the CRP level and ESR may help appropriately assess the disease activity and guide decisions about immunosuppressive therapy for BD. A retrospective evaluation of this course suggests that we should have considered discontinuing anticoagulants before initiating immunosuppressive therapy.

In conclusion, the possibility of BD should be considered for patients with history of recurrent hemoptysis and venous thrombosis. For BD patients with major vessel thrombosis and PAA, great care should be taken when administering anticoagulants to prevent bleeding events and control systemic inflammation, which can be the pathogenesis of vascular complications in BD.

Our institutional review board approved the study, and we obtained written informed consent from the family of the patient.

The authors state that they have no Conflict of Interest (COI).

\section{References}

1. Bousser MG, Ferro JM. Cerebral venous thrombosis: an update. Lancet Neurol 6: 162-170, 2007.

2. Tayer-Shifman OE, Seyahi E, Nowatzky J, Ben-Chetrit E. Major vessel thrombosis in Behçet's disease: the dilemma of anticoagulant therapy-the approach of rheumatologists from different countries. Clin Exp Rheumatol 30: 735-740, 2012.

3. Emmi G, Silvestri E, Squatrito D, et al. Thrombosis in vasculitis: from pathogenesis to treatment. Thromb J 13: 15, 2015.

4. Pengo V, Denas G, Zoppellaro G, et al. Rivaroxaban vs warfarin in high-risk patients with antiphospholipid syndrome. Blood 132: 1365-1371, 2018.

5. Mader R, Ziv M, Adawi M, Mader R, Lavi I. Thrombophilic factors and their relation to thromboembolic and other clinical manifestations in Behçet's disease. J Rheumatol 26: 2404-2408, 1999.

6. Saadoun D, Wechsler B, Resche-Rigon M, et al. Cerebral venous 
thrombosis in Behçet's disease. Arthritis Rheum 61: 518-526, 2009.

7. Ahn JK, Lee YS, Jeon CH, Koh EM, Cha HS. Treatment of venous thrombosis associated with Behçet's disease: immunosuppressive therapy alone versus immunosuppressive therapy plus anticoagulation. Clin Rheumatol 27: 201-205, 2008.

8. Desbois AC, Wechsler B, Resche-Rigon M, et al. Immunosuppressants reduce venous thrombosis relapse in Behçet's disease. Arthritis Rheum 64: 2753-2760, 2012.

9. Alibaz-Oner F, Karadeniz A, Ylmaz S, et al. Behçet disease with vascular involvement: effects of different therapeutic regimens on the incidence of new relapses. Medicine (Baltimore) 94: e494, 2015.

10. Sarica-Kucukoglu R, Akdag-Kose A, KayabalI M, et al. Vascular involvement in Behçet's disease: a retrospective analysis of 2319 cases. Int J Dermatol 45: 919-921, 2006.

11. Uzun O, Akpolat T, Erkan L. Pulmonary vasculitis in Behçet disease: a cumulative analysis. Chest 127: 2243-2253, 2005.

12. Seyahi E, Yurdakul S. Behcet's syndrome and thrombosis. Mediterr J Hematol Infect Dis. Forthcoming.

13. Hatemi G, Christensen R, Bang D, et al. 2018 update of the EULAR recommendations for the management of Behçet's syndrome. Ann Rheum Dis 77: 808-818, 2018.

14. Calamia KT, Schirmer M, Melikoglu M. Major vessel involvement in Behçet's disease: an update. Curr Opin Rheumatol 23: 24-31, 2011.

15. Konstantinides SV, Torbicki A, Agnelli G, et al. 2014 ESC guidelines on the diagnosis and management of acute pulmonary embolism. Eur Heart J 35: 3033-3069, 2014.

16. Kearon C, Akl EA, Comerota AJ, et al. Antithrombotic therapy for VTE disease: antithrombotic therapy and prevention of thrombosis, 9th ed: American College of Chest Physicians evidence-based clinical practice guidelines. Chest 141: e419S-e494S, 2012.

17. Klok FA, Hösel V, Clemens A, et al. Prediction of bleeding events in patients with venous thromboembolism on stable anticoagulation treatment. Eur Respir J 48: 1369-1376, 2016.

18. Yagi T, Yamagishi F, Mizutani F, et al. A case of Behçet's disease presenting with Hughes-Stovin syndrome (multiple pulmonary arterial aneurysms remitting with corticosteroid therapy. Nihon Kokyuki Gakkai Zasshi 39: 140-144, 2001 (in Japanese, Abstract in English).

19. Erkan D, Yazici Y, Sanders A, Trost D, Yazici H. Is HughesStovin syndrome Behçet's disease? Clin Exp Rheumatol 22: S64S68, 2004.

20. Hamuryudan V, Oz B, Tüzün H, Yazici H. The menacing pulmonary artery aneurysms of Behçet's syndrome. Clin Exp Rheumatol 22: S1-S3, 2004.

21. Tüzün H, Hamuryudan V, Yildirim S, et al. Surgical therapy of pulmonary arterial aneurysms in Behçet's syndrome. Ann Thorac Surg 61: 733-735, 1996.

22. Bradbury AW, Milne AA, Murie JA. Surgical aspects of Behçet's disease. Br J Surg 81: 1712-1721, 1994.

23. Herb S, Hetzel M, Hetzel J, Friedrich J, Weber J. An unusual case of Hughes-Stovin syndrome. Eur Respir J 11: 1191-1193, 1998.

24. Mahlo HR, Elsner K, Rieber A, Brambs HJ. New approach in the diagnosis of and therapy for Hughes-Stovin syndrome. AJR 167: 817-818, 1996.

25. Erkan F. Pulmonary involvement in Behçet's Disease. Curr Opin Pulm Med 5: 314-318, 1999.

26. Tunaci M, Ozkorkmaz B, Tunaci A, Gül A, Engin G, Acunaş B. CT findings of pulmonary artery aneurysms during treatment for Behçet's disease. AJR 172: 729-733, 1999.

27. Gaitonde S, Samols D, Kushner I. C-reactive protein and systemic lupus erythematosus. Arthritis Rheum 59: 1814-1820, 2008.

28. Kay J, Morgacheva O, Messing SP, et al. Clinical disease activity and acute phase reactant levels are discordant among patients with active rheumatoid arthritis: acute phase reactant levels contribute separately to predicting outcome at one year. Arthritis Res Ther 16: R40, 2014.

The Internal Medicine is an Open Access journal distributed under the Creative Commons Attribution-NonCommercial-NoDerivatives 4.0 International License. To view the details of this license, please visit (https://creativecommons.org/licenses/ by-nc-nd/4.0/).

(C) 2020 The Japanese Society of Internal Medicine Intern Med 59: 1615-1620, 2020 